\title{
SNP identification, verification, and utility for population genetics in a non-model genus
}

\author{
Larissa M Williams ${ }^{1}$, Xin Ma², Adam R Boyko², Carlos D Bustamante² and Marjorie F Oleksiak*3
}

\begin{abstract}
Background: By targeting SNPs contained in both coding and non-coding areas of the genome, we are able to identify genetic differences and characterize genome-wide patterns of variation among individuals, populations and species. We investigated the utility of 454 sequencing and MassARRAY genotyping for population genetics in natural populations of the teleost, Fundulus heteroclitus as well as closely related Fundulus species (F. grandis, F. majalis and $F$. similis).
\end{abstract}

Results: We used 454 pyrosequencing and MassARRAY genotyping technology to identify and type 458 genome-wide SNPs and determine genetic differentiation within and between populations and species of Fundulus. Specifically, pyrosequencing identified 96 putative SNPs across coding and non-coding regions of the F. heteroclitus genome: 88.8\% were verified as true SNPs with MasSARRAY. Additionally, putative SNPs identified in F. heteroclitus EST sequences were verified in most (86.5\%) F. heteroclitus individuals; fewer were genotyped in F. grandis (74.4\%), F. majalis (72.9\%), and F. similis (60.7\%) individuals. SNPs were polymorphic and showed latitudinal clinal variation separating northern and southern populations and established isolation by distance in F. heteroclitus populations. In F. grandis, SNPs were less polymorphic but still established isolation by distance. Markers differentiated species and populations.

Conclusions: In total, these approaches were used to quickly determine differences within the Fundulus genome and provide markers for population genetic studies.

\section{Background}

High throughput sequencing and genotyping has become increasingly faster, less expensive and more accurate. In recent years this has lead to the establishment of myriad data sets ranging from increased coverage of variation in the human genome at the individual level [1-5] to the sequencing of non-model prokaryotic and eukaryotic genomes and transcriptomes [6-11]. For many organisms sequencing of entire genomes is still unattained, but smaller, more targeted portions of the genome can be easily sequenced and genotyped. Such data can provide genome-wide sequence information which can be used to characterize population and selection pressure parameters as well as provide evolutionary insights that are broadly applicable [12].

One non-model genus, Fundulus, includes closely related species that range in physiology, environmental

* Correspondence: moleksiak@rsmas.miami.edu

${ }^{3}$ Rosenstiel School of Marine and Atmospheric Sciences, University of Miami, 4600 Rickenbacker Causeway, Miami, FL 33149, USA

Full list of author information is available at the end of the article and habitat preference, and geographic locales; Fundulus heteroclitus and Fundulus majalis inhabit the Atlantic coast, and Fundulus grandis and Fundulus similis inhabit the Gulf Coast. Many Fundulus species and/or populations have extensive euryhaline capabilities, respond well to varying ranges of hypoxia [13-15], live along a steep thermocline, and have adapted to extremely polluted areas [16]. A variety of studies have investigated the underlying genetic basis of this teleosts' phenotypic plasticity. While some of the transcriptome is known for $F$. heteroclitus [17-27] much of the genome-wide variation within and between populations and species for this genus is relatively unknown.

Establishing a set of genetic markers, which can be used to assess regions of the genome involved in local adaptation and in speciation is important to understand fundamental similarities and differences between populations and species of Fundulus. Once markers are established they can be further studied to look for signatures of selection to any number of evolutionary forces (e.g., pollution, hypoxia, salinity, temperature). A few studies have estab- 
lished genetic differences between populations of $F$. heteroclitus mainly with respect to phylogeographic constraints [28,29] or selection [30-38]. These studies used microsatellite, mitochondrial DNA, and AFLP analyses as well as targeted gene approaches. Single nucleotide polymorphisms (SNPs) are a useful starting point to scan large and disparate regions of the genome due to their abundance in both coding and non-coding regions, their co-dominant nature, and lack of ambiguity.

SNPs have been used to establish differences between individuals [39], populations [40-42] and species [43,44]. They also are useful markers for propensity to disease [45-47], disease states [48], and evidence of the genetic basis of adaptation [49-52]. In vertebrates, a SNP occurs on average every 100 to 1000 base pairs and often is in linkage disequilibrium with many other SNPs along the chromosome, forming strong haplotypes, which can be easily identified [53]. Unfortunately, SNP resources are not readily available in the majority of non-model species lacking genomic resources. With this in mind, we set out to establish a set of SNP markers to identify differences between Fundulus populations and species.

\section{Methods}

\section{Sample Collection and Extraction}

$F$. heteroclitus were collected using minnow traps during the spring of 2005. Spleen and testes were sampled from 20 individuals from each of ten collection sites along the East coast of the United States (Figure 1). F. grandis were collected using minnow traps during the winter of 2009 (Figure 1). Fin clips were sampled from 15 individuals from each of the six collection sites along the Gulf Coast of the United States. Spleen from F. majalis was extracted from 13 individuals from Woods Hole, Massachusetts and 10 individuals from Sapelo Island, GA. Spleen also was extracted from $F$. similis collected from Pensacola, Florida (3 individuals) and Corpus Christi, Texas (8 individuals).

Genomic DNA from spleen and testes was extracted by phenol and chloroform as described in Wirgin et al. [54], and DNA was resuspended in $50 \mu \mathrm{L} 0.1 \times$ TE buffer. Genomic DNAs from fin clips were extracted using a modified version of Aljanabi and Martinez [55] and DNA was resuspended in $50 \mu \mathrm{L} 0.1 \times \mathrm{TE}$ buffer. This experiment was performed according to an approved Institutional Animal Care and Use Committee at North Carolina State University.

\section{DNA Pyrosequencing}

F. heteroclitus genomic DNAs (500 ng) from eight individuals in each of ten collection sites (all sites except Point Judith, RI, Figure 1A) were digested individually with 1 U BspE1 (New England Biolabs, MA) and $1 \mathrm{U}$ EcoRI (New England Biolabs, MA). Samples were incu- bated for three hours at $37^{\circ} \mathrm{C}$ in a total volume of $30 \mu \mathrm{L}$ containing Buffer 3 (New England Biolabs, MA). Adaptors (Table 1) to each of the restriction sites, $25 \mathrm{mM}$ ATP, and $1 \mathrm{U}$ of T4 DNA ligase (Epicentre) were added to reactions and incubated at $16^{\circ} \mathrm{C}$ overnight. A 2' O-methyl block was added to the $3^{\prime}$ cytosine base on the adapter. This block assured that only those fragments digested with both BspEI and EcoRI would be amplified with PCR and prevented amplification of fragments with the same type of restriction site on both ends of the fragment.

Preselective PCR reactions with primers specific to adaptors (Table 1 ) were performed in a total volume of 25 $\mu \mathrm{L}$ containing $2 \mu \mathrm{L}$ of diluted (1:10 in $0.1 \times$ Tris-EDTA buffer) ligation product with EcoRI primer (Integrated DNA Technologies; 10 pmol), BspE1 primer (Integrated DNA Technologies; $10 \mathrm{pmol}$ ) and $1 \mathrm{U}$ Taq. PCR conditions were 20 cycles of $94^{\circ} \mathrm{C}$ for $10 \mathrm{sec}, 49^{\circ}$ for $30 \mathrm{sec}$, and $72^{\circ} \mathrm{C}$ for one min. Following the preselective amplification, a selective amplification was carried out to decrease the number of fragments amplified in each individual to approximately 200 by extending the primer on the 3 ' end. Preselective PCR products were diluted (1:10) and $2 \mu \mathrm{L}$ of diluted product was amplified with primers (Table 1) to EcoRI+ AAG (Integrated DNA Technologies; 10 pmol) and BspEI $+\mathrm{C}$ (Integrated DNA Technologies; 10 pmol) with $1 \mathrm{U} \mathrm{Taq}$ in a $25 \mu \mathrm{L}$ total volume. PCR conditions in the first cycle were $94^{\circ} \mathrm{C}$ for $10 \mathrm{sec}, 65^{\circ} \mathrm{C}$ for $30 \mathrm{sec}$, and $72^{\circ} \mathrm{C}$ for one minute with the annealing temperature reduced by $0.5^{\circ} \mathrm{C}$ for 20 cycles, then 25 cycles of $94^{\circ} \mathrm{C}$ for $10 \mathrm{sec}, 55^{\circ} \mathrm{C}$ for $30 \mathrm{sec}$, and $72^{\circ} \mathrm{C}$ for one minute.

Primers (Table 1) specific to the EcoRI restriction site were generated with the goals of labeling the DNA fragments from each individual with specific nucleotide barcodes [56] and preparing those samples for emulsionbased amplification. Starting at the 5 ' end, 19 nucleotides (Table 1) complementary to the primer on the DNA capture beads used in the emulsion PCR reaction [57] were synthesized (Integrated DNA Technologies). Following those nucleotides, each primer had a distinct 10 base pair barcode [56] used to identify individuals (ten primers in total). The final 19 base pairs of the primer were specific to the EcoRI adapter. The BspE1 primer (Table 1) started at its $5^{\prime}$ end with 19 nucleotides (Table 1), which were complementary to the primer on the DNA capture beads followed by 18 base pairs specific to the BspE1 adapter (Figure 2). All primers were HPLC purified. Amplified selective fragments were diluted (1:10) and added to both EcoRI and BspE1 primers (Integrated DNA Technologies; $10 \mathrm{pmol}$ ) in a $25 \mu \mathrm{L}$ volume. PCR conditions were $94^{\circ} \mathrm{C}$ for $10 \mathrm{sec}, 50^{\circ} \mathrm{C}$ for $30 \mathrm{sec}$, and $72^{\circ} \mathrm{C}$ for one minute and were carried out for 30 cycles. PCR reactions were pooled into eight wells, where each of the ten distinct barcodes was represented only once in each of the pools. Each pool of PCR products was purified using QIAquick PCR Puri- 


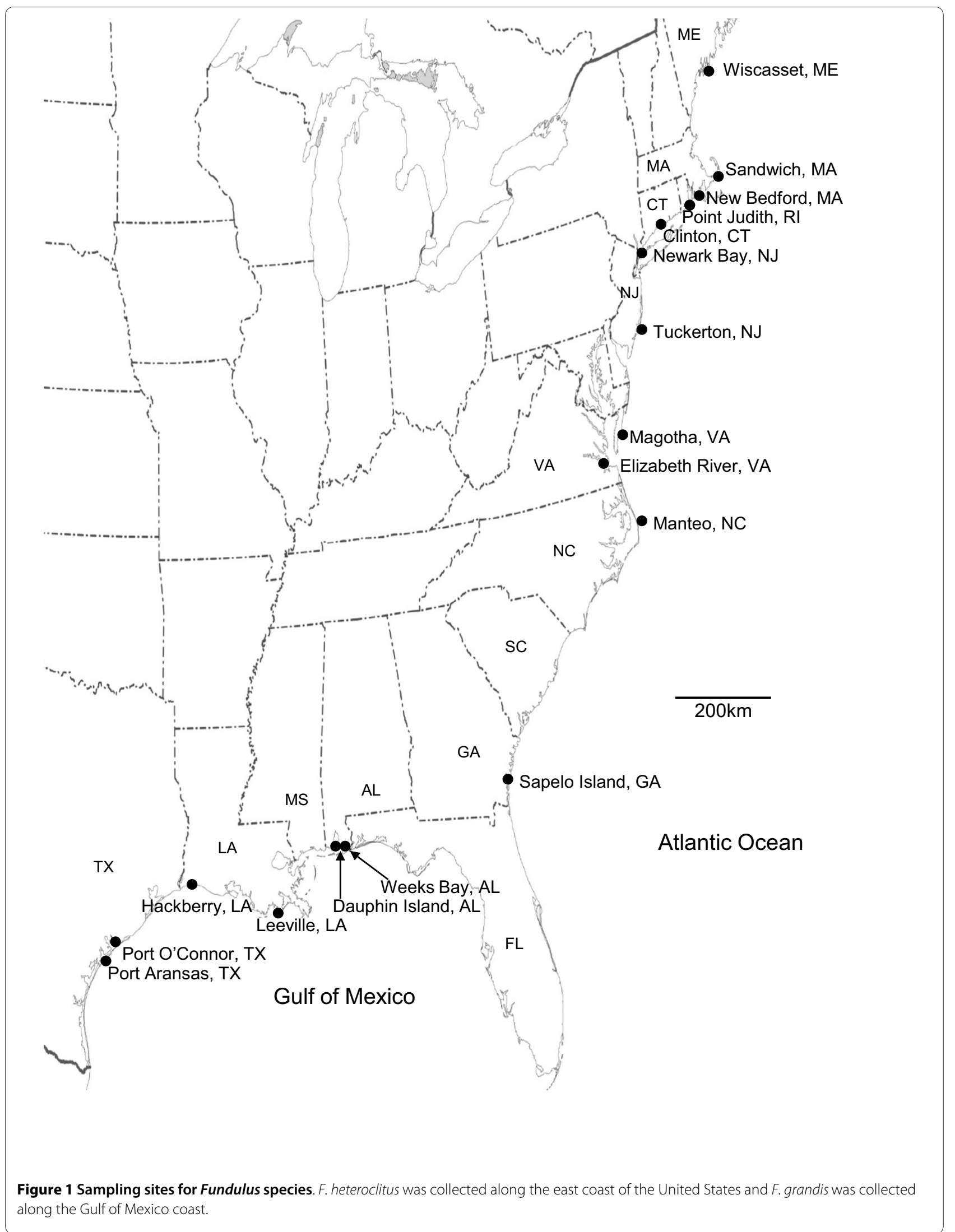


Table 1: Adapters and primers used in the amplification of genomic DNA.

Adapters

BspEl (5' to 3')

GACGATGAGTCCTGAGC

CTGCTACTCTCAGGACTCGGGCC

EcoRI (5' to 3')

CTAGAGTCCTAGTAGCACCTCGTAGACTGCGTACC

*CATCTGACGCATGGTTAA

Preselective Primers

EcoRI (5' to 3')

CTGAGTCCTAGTAGCACC

BspEl (5' to 3')

GACGATGAGTCCTGAGC

Selective Primers

EcoRI (5' to 3')

GACTGCGTACCAATTCAAG

BspEl (5' to 3')

GACGATGAGTCCTGAGCC

Barcoded Primers

EcoRI (5' to 3')

1 GCCTCCCTCGCGCCATCAGAGCCTAAGCTGACTGCGTACCAATT CAAG

2 GCCTCCCTCGCGCCATCAGAGTTCAAGTCGACTGCGTACCAATT CAAG

3 GCCTCCCTCGCGCCATCAGACTTGAACTGGACTGCGTACCAATT CAAG
Table 1: Adapters and primers used in the amplification of genomic DNA. (Continued)

4 GCCTCCCTCGCGCCATCAGACGGTAACGTGACTGCGTACCAATT CAAG

5 GCCTCCCTCGCGCCATCAGATCCGAATCGGACTGCGTACCAATT CAAG

6 GCCTCCCTCGCGCCATCAGATGGCAATGCGACTGCGTACCAATT CAAG

7 GCCTCCCTCGCGCCATCAGCAGGTCCAGTGACTGCGTACCAATT CAAG

8 GCCTCCCTCGCGCCATCAGCATTGCCATGGACTGCGTACCAATT CAAG

9 GCCTCCCTCGCGCCATCAGCTAAGCCTAGGACTGCGTACCAATT CAAG

10 GCCTCCCTCGCGCCATCAGCGAATCCGATGACTGCGTACCAATT CAAG

BspEl (5' to 3')

GCCTTGCCAGCCCGCTCAGGACGATGAGTCCTGAGCC

*Star indicates location of 2' O-methyl block.

fication Kit (Qiagen, USA). PCR products were further purified with AMPure (Agencourt).

Emulsion PCR was carried out on PCR products as described [57]. Amplification of the PCR product on the bead was controlled for by quantifying and calculating the size of the amplicon pool using a Bioanalyzer 2100 so that there was a minimum of $2 \times 10^{6}$ copies of DNA that ranged in size from 100 to 700 base pairs. Subsequent products were sequenced on a Roche/454 Life Sciences GS FLX Sequencer at the University of South Carolina's Environmental Genomics Core Facility. The PicoTiter plate was subdivided into eight regions with an expectation of 30,000 reads per region [58].

Assembly of pyrosequencing sequences and SNP Detection Sequences were trimmed of their barcodes. All 626 sequences with at least one ambiguous base were removed since the presence of even a single ambiguous base is an effective indicator of low-quality sequence [59]. Because shorter than expected read lengths also correlate strongly with incorrect reads [60], another three percent of the sequences (whose lengths were smaller than 100 $\mathrm{bp)}$ were removed. The remaining reads were aligned using CAP3 [61]. Quality scores were rescaled to be comparable to the usual Phred Score using ARACHNE [62].

SNPs were called at both the individual level and population level. At the individual level, SNPs were called using both a Bayesian method and a likelihood ratio test (LRT) method. For the Bayesian method, 10-4 was used as 


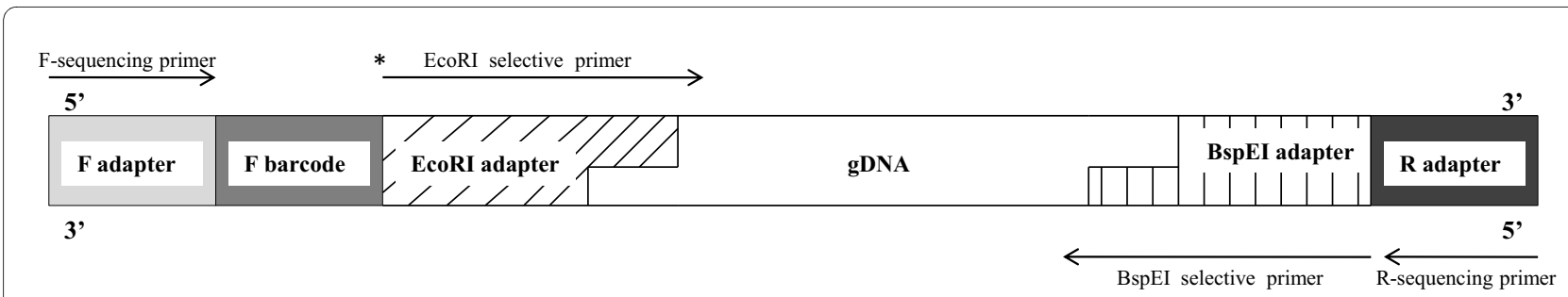

Figure 2 Design of 454 pyrosequencing contig generated from the digestion of genomic DNA with restriction enzymes (EcoRI and BspEI), the addition of restriction site specific linkers, an individual barcode and a 454 amplicon adapter.

the prior for the mutation rate [63]. At the population level, for each locus on the contig, we simulated the error model and marked a locus as a potential SNP if it had a larger number of second alleles in comparison to the critical value from the error model. Furthermore, a potential SNP site had to have at least three individuals sequenced to $2 \times$ at that locus unless another potential SNP site was within five basepairs or over $90 \%$ of the individuals had been classified as heterozygous at the individual level. This was done to minimize the rate of false positives caused by homologs.

\section{Bayesian and LRT model for SNP calling at individual level}

For the Bayesian model, for each contig, Prior $=1 \times 10^{-4}$ represents the mutation rate; $N$ represents the total number of unique mapping loci with multiple allelic types; $A^{i}$ and $a^{i}$ represent, respectively, the major and minor alleles at locus $i ; N_{i}$ represents the total number of alleles observed for locus $\mathrm{i}$, and $Y_{j}$ is the type of the $j^{\text {th }}$ allele copy among these $N_{i}$ alleles where $j=0 \cup N_{i}$; finally, $e_{j}$ is the probability of error of the $j^{\text {th }}$ allele where the error probability is computed as $10^{\frac{-Q}{10}}$ and where $Q$ is the corresponding quality score after rescaling.

The posterior probability for the $i^{\text {th }}$ locus being homozygous or heterozygous is:

$$
\begin{aligned}
& \mathbf{P}(\text { Hetero. } \mid \text { data })=\frac{\mathbf{P}(\text { data } \mid \text { Hetero. }) \times \mathbf{P}(\text { Hetero. })}{\mathbf{P}(\text { data })} \\
& \sim \mathbf{P}(\text { data } \mid \text { hetero. }) \times \mathbf{P}(\text { Hetero. }) \\
& \sim \text { Prior } \times(0.5)^{N i} \\
& \mathbf{P}(\text { Homo. } \mid \text { data })=\frac{\mathbf{P}(\text { data } \mid \text { Homo. }) \times \mathbf{P}(\text { Homo. })}{\mathbf{P}(\text { data })} \\
& \sim \mathbf{P}(\text { data } \mid \text { hom o. }) \times \mathbf{P}(\text { Homo. }) \\
& \sim(1-\text { Prior }) \times \prod_{j}^{N i}\left(1-e_{j}\right)^{1\left(Y_{j}=A^{i}\right)} \times e_{j}^{1\left(Y_{j}=a^{i}\right)}
\end{aligned}
$$

Based on the posterior probabilities from above, we classified each of these $\mathrm{N}$ loci as homozygous or heterozygous exclusively. If a locus was classified as heterozygous, it was further tested using a likelihood ratio test (LRT) as follows:

For a particular locus $i$ on the contig:

$$
\begin{aligned}
& \mathbf{P}\left(X_{i}=A^{i}\right)=p \\
& \mathbf{P}\left(X_{j}=a^{i}\right)=1-p
\end{aligned}
$$

where $X_{j}$ stands for the true allele that we should have observed. For each $Y_{j}$, we have an error probability of $e_{j}$ associated with it.

Then we have:

$$
\begin{aligned}
& \mathbf{P}\left(Y_{i}=A^{i} \mid X_{i}=A^{i}\right)=1-e_{j} \\
& \mathbf{P}\left(Y_{i}=a^{i} \mid X_{i}=a^{i}\right)=1-e_{j} \\
& \mathbf{P}\left(Y_{i}=a^{i} \mid X_{i}=A^{i}\right)=e_{j} \\
& \mathbf{P}\left(Y_{i}=A^{i} \mid X_{i}=a^{i}\right)=e_{j}
\end{aligned}
$$

Therefore we have:

$$
\begin{aligned}
& \mathrm{P}\left(Y_{i}=A^{i} \mid X_{i}=A^{i}\right)=\left(1-e_{j}\right) \times p+e_{j} \times(1-p) \\
& \mathrm{P}\left(Y_{i}=a^{i} \mid X_{i}=A^{i}\right)=e_{j} \times p+\left(1-e_{j}\right) \times(1-p)
\end{aligned}
$$

and

$$
Y_{j} \sim \text { Bernoulli }\left(1,\left(1-e_{j}\right) \times p+e_{j} \times(1-p)\right)
$$

Based on all of the above, the likelihood of locus $I$ was computed as:

$L=\prod_{j=1}^{N_{j}}\left\{\left(1-e_{j}\right) \times p+e_{j} \times(1-p)\right\}^{I_{j}}\left\{e_{j} \times p+\left(1-e_{j}\right) \times(1-p)\right\}^{1-I_{j}}$ 
Where $I_{j}=1$ if $Y_{j}=A_{i}$; and $I_{j}=0$ if $Y_{j}=a_{i}$

The LRT was performed with the hypothesis of $H_{O}: p=$ 0.5 versus $H_{a}: p>0.5$ and $-2 \times L R T \sim X^{2}(1)$.

\section{Error model simulating}

In order to call SNPs at the population level, we simulated the error model for each locus with multiple allelic types; we assumed that a particular locus was homozygous with major allele $A^{i}$ and randomly simulated $N_{i}$ number of alleles copies to be $A^{i}$ or any of the other three allele types from a uniform distribution with probability $\left(1-e_{j}\right)$ and $e_{j}$ respectively. We repeated this process 10,000 times and recorded the different numbers of second alleles found in the simulation. The critical value was chosen as the number of second alleles with a right-side p-value of 0.001 .

\section{Validation of SNPs}

Multiplex assays targeting 458 SNPs in 250 F. heteroclitus individuals, $90 \mathrm{~F}$. grandis individuals, $23 \mathrm{~F}$. majalis individuals, and $21 F$. similis individuals were attempted using the Sequenom MassARRAY technology. These consisted of 81 putative SNPs identified by the $F$. heteroclitus pyrosequencing, 350 putative SNPs previously identified in F. heteroclitus ESTs [64], and 27 putative SNPs from 22 genes containing, amongst others, SNPs in the aryl hydrocarbon receptor [65], lactate dehydrogenase B [29], and the proximal promoter of cytochrome P4501A (unpublished). Assays were designed using the MassARRAY Assay Design Software with the goal of maximizing multiplexing of 36 SNPs per well (Sequenom, San Diego, CA, USA). Only SNPs where 70 base pairs were annotated on either side of the polymorphism were included in the study. There were 14 SNPs previously identified with 454 pyrosequencing where this criterion was not met. If multiple SNPs were proximal ( $<70$ base pairs) to one another, one SNP was chosen and the other(s) was translated into a degenerate nucleotide (e.g., $\mathrm{K}=\mathrm{G}$ or $\mathrm{T}$ ). Reaction conditions were performed by iPLEX chemistry as recommended by Sequenom across 13 plates at the University of Minnesota's BioMedical Genomics Center. SNP genotypes were called using the Sequenom System Typer 4.0 Analysis package. This software uses a threeparameter model to calculate the significance of each putative genotype. Based on the relative significance, a final genotype is called and assigned a particular name (e.g., conservative, moderate, aggressive, user call). Noncalls also were noted (e.g., low probability, bad spectrum).

\section{Analysis of Genotype Data}

Arlequin v.3.11 was used to calculate genetic diversity among populations (of F. heteroclitus and F. grandis) by calculating the percentage of polymorphic SNPs $\left(P_{O}\right)$, observed $\left(H_{O}\right)$ and expected heterozygosity $\left(H_{E}\right)$, and the within-population fixation index $(F)$ [66]. Fixation index deviations from zero were tested by 10,000 permutations of alleles between individuals. Hardy-Weinberg equilibrium also was tested in each population. An analysis of molecular variance (AMOVA) was performed to calculate the distribution of variance within populations, between North and South regions, and between F. heteroclitus populations within North and South regions. For $F$. grandis, the AMOVA was performed to calculate the distribution of variance within populations as well as between populations longitudinally along the Gulf of Mexico. Since SNPs were initially identified from $F$. heteroclitus sequence data, a maximum of $5 \%$ missing data was used as a parameter for calculations involving $F$. heteroclitus and $10 \%$ for all others.

A Mantel test was performed to assess the assumption of isolation by distance using XLSTAT 2009 for F. heteroclitus and F. grandis.

STRUCTURE v.2.2 [67,68] was used to estimate the number of populations (K) in F. heteroclitus, F. grandis, $F$. majalis and F. similis along both the Western Atlantic and the Gulf of Mexico and to assign individuals to these populations. The Monte Carlo Markov Chain was run for $10^{5}$ iterations following a burn-in period of $10^{5}$ iterations for $\mathrm{K}=1$ to 14 using the correlated allele frequencies model and assumed admixture. Distruct v. 1.1 [69] was used to generate bar plots to depict classifications with the highest probability under the model. JMP Genomics 3.2 for SAS 9.1.3 conducted principal component analysis on all samples to establish population structure.

\section{Results}

\section{GS FLX Sequencing and Assembly}

A total of 111,001 reads were obtained in one run of the GS FLX instrument producing 5,346,445 total bases of sequence (average read length of 218 bases) with $99.98 \%$ of bases having a quality score of 20 or greater. Across the eight regions of the plate, there were on average 1,982 reads per individual. The third barcode produced many less reads per region $(<1,000)$ amongst all regions. All other barcodes performed very similarly with respect to the number of reads per individual across regions. Only $46 \%$ of the number of expected reads $(111,001$ instead of $240,000)$ were obtained from sequencing. Prior to sequencing, the amplification success of loci on the beads was checked for quality using a Bioanalyzer 2100, and all samples passed. However, three of the eight regions produced half the expected number of reads and a fourth region produced only $15 \%$ the expected number of reads. This indicated local problems in sequencing with respect to particular regions and the samples in those regions rather than the plate as a whole. All control beads passed the filter control with an average percentage of $90 \%$ across all regions, whereas the percentage of samples passing the filter control varied between regions and 
averaged 36\%: regions with fewer than expected reads had fewer samples passing the filter control. Two regions had very high failure rates due to mixed samples, indicating more than one amplicon per bead.

Upon alignment 1,464 contigs were obtained with an average length of 213 base pairs. The average coverage across all loci was 22 reads per contig (Figure 3). Due to the low coverage of any one contig per individual, the detection of a SNP within a contig was mainly based on its presence across populations rather than at the individual level. Of the 1,464 contigs obtained, 96 contained SNPs. Within these contigs, 261 SNPs were identified. Among those contigs containing SNPs, the average length was 243 base pairs with an average coverage of 184 reads per contig (Figure 3). The observed rate of SNP detection is a function of depth, so as read counts per contig increased so did the number of SNPs detected. One third of all contigs with identified SNPs had only one SNP and $57 \%$ had two or fewer SNPs per contig. SNPs were distributed approximately evenly along the position in the contig $\left(\mathrm{R}^{2}=0.01\right)$.

\section{Genotyping success}

Of the initial 458 loci we attempted to amplify, 277 had a greater than $90 \%$ successful call rate among all individuals with no more than two alleles per SNP. In F. heteroclitus $61.4 \%$ of all loci amplified in greater than $95 \%$ of individu- als. In F. grandis, $25.6 \%$ of SNPs did not amplify, and $58.2 \%$ of SNPs were monomorphic (Table 2). $24 \%$ of the monomorphic SNPs in F. grandis also were monomorphic in $F$. heteroclitus, but for the alternative allele, indicating fixed differences between the two species.

On average, $80 \%$ (SD $= \pm 7.4 \%$ ) of the putative SNPs identified with 454 pyrosequencing were amplified with MassARRAY in F. heteroclitus individuals: 72 of the 81 loci $(88.8 \%)$ were polymorphic, 8 loci $(9.8 \%)$ were monomorphic, and one locus did not amplify. Among all other putative SNPs genotyped with MassARRAY, 83\% were successfully amplified. However, $13.5 \%$ of all loci in $F$. heteroclitus, $25.6 \%$ in F. grandis, $27.1 \%$ in F. majalis and $39.3 \%$ in $F$. similis did not amplify (Figure 4a). Many nonheteroclitus loci were also not polymorphic, and in $F$. heteroclitus $12.3 \%$ of all loci were monomorphic, as were $58.2 \%$ in F. grandis, $26.4 \%$ in F. majalis, and $29.7 \%$ in $F$. similis (Figure 4b). Due to the divergence between species resulting in unsuccessful amplification in non-heteroclitus individuals, locus amplification success was addressed on a species and population level for all remaining tests and not on the overall amplification success. Due to the low sample size, amplification rate, and predominant monomorphism of loci in F. majalis and F. similis samples, further characterizations of genetic parameters (with the exception of population structure) were not carried out for these two species.

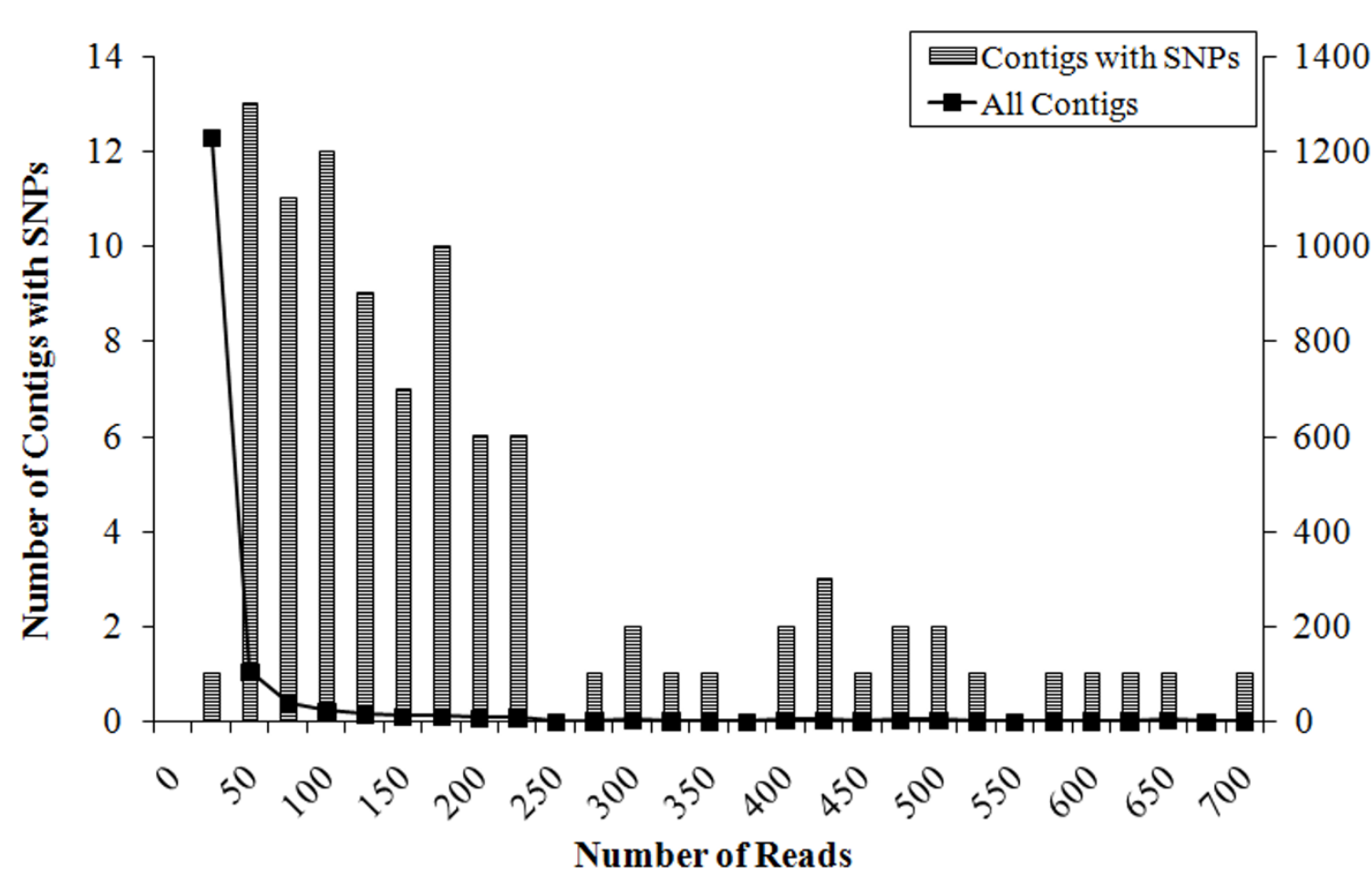

Figure 3 Contig totals versus number of reads per contig amongst those contigs with identified SNPs (bars) and all contigs (squares) 
Table 2: Genotyping success of SNP markers using the MASSARRAY multiplex assay.

\begin{tabular}{lcc}
\hline Category & Number of SNPs & Percentage of SNPs \\
\hline SNPs called in $>95 \%$ of $F$. heteroclitus individuals & 259 & 61.4 \\
SNPs called in $<80 \%$ of all individuals & 135 & 31.9 \\
SNPs called in $>90 \%$ but $<95 \%$ of all individuals & 101 & 23.9 \\
Monomorphic SNPs called in $>95 \%$ of all individuals & 23 & 5.4 \\
Polymorphic SNPs called in $>95 \%$ of all individuals & 163 & 38.6 \\
SNPs called in $<90 \%$ of all individuals identified in 454 & 35 & 43.2 \\
SNPs called in $>90 \%$ of all individuals identified in 454 & 46 & 56.8 \\
\hline
\end{tabular}

SNPs which were identified by Sequenom software as low probability in greater than $50 \%$ of all individuals were removed (17 SNPs in total). An additional 20 SNPs were excluded from analyses due to their excessive heterozygosity across individuals and populations of $F$. heteroclitus. These SNPs may represent segmental duplication where the two duplicate regions are identical, except that a SNP has been driven to high frequency or become fixed in one of the duplicates.

\section{Genetic Diversity}

The percentage of polymorphic SNPs $\left(P_{O}\right)$ ranged from $3.7 \%$ to $67 \%$ (Table 3 ) among populations and species. The percentages of polymorphic SNPs were significantly different between northern and southern populations of F. heteroclitus where levels decreased in populations further north and east $(\mathrm{p}=0.035)$. Among F. grandis populations, the percentages of polymorphic SNPs did not significantly differ along latitude $(\mathrm{p}=0.143)$ or longitude $(\mathrm{p}=0.415)$. Among populations, most loci were in HardyWeinberg equilibrium (Table 3). Observed heterozygosity $\left(H_{O}\right)$ among all populations ranged from 0.016 to 0.17 with a mean of 0.10 (Table 3). Observed heterozygosity was lower in northern $F$. heteroclitus in comparison to

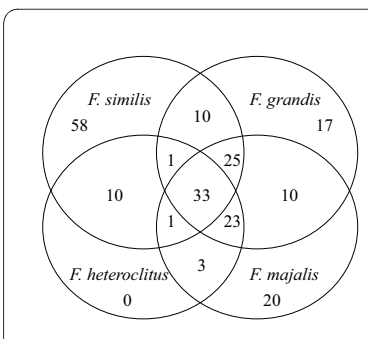

(A)

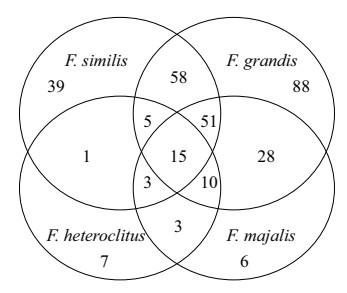

(B)
Figure 4 Non-amplified and non-polymorphic loci among Fundulus species. (A) Numbers of loci, which did not amplify with the MassARRAY platform among the four species of Fundulus. Not shown: loci shared between F. majalis and F. similis (8) and F. heteroclitus and F. grandis (12). (B) Numbers of loci, which were not polymorphic among the four species. Not shown: loci shared between F. majalis and F. similis (9) and F. heteroclitus and F. grandis (1). southern populations $(\mathrm{p}=0.04)$ and did not differ along latitude $(\mathrm{p}=0.72)$ or longitude $(\mathrm{p}=0.33)$ in $F$. grandis. Average expected heterozygosity $\left(H_{E}\right)$ ranged from 0.019 to 0.20 with a mean of 0.11 (Table 3 ). The average withinpopulation fixation index, $F$, averaged over all polymorphic loci was on average 0.16 in $F$. heteroclitus and 0.20 in F. grandis (Table 3).

SNPs identified via 454 sequencing did not have genetic parameters that differed from SNPs identified in ESTs with the exception of Hardy-Weinberg equilibrium. 454-derived SNPs had a higher percentage of SNPs not in Hardy-Weinberg equilibrium due to a lack of heterozygosity (22\% versus $9 \%$ ).

Many SNP loci (60\%) in F. heteroclitus had a frequency greater than 0.10 and were considered common SNPs (Additional file 1). In contrast, $90 \%$ of SNPs in F. grandis had low minor allele frequencies below 0.10 .

\section{Population Structure}

The two independent tests of population stratification (STRUCTURE and principle component analysis (PCA)) identified species and population differences in all samples (Figure 5). STRUCTURE analysis, which uses a Bayesian MCMC clustering approach to assign individuals to clusters, separated populations into eight different clusters $(\operatorname{Pr}(K)=0.37$; Figure 4a). At the most probable clustering of the data $(K=8)$, ten runs produced nearly identical membership coefficients which had pairwise similarity coefficients greater than 0.98 . F. heteroclitus clustered north to south and $F$. grandis as its own separate cluster. Among F. heteroclitus, individuals from Maine and Georgia, the most northern and southern collection sites, formed their own distinct clusters. Individuals from sites between Maine and Georgia clustered with others from geographically similar sites. F. majalis and F. similis clustered together and away from the other two species. Similarly, in the PCA analysis, which does not rely on modeling the data, northern and southern $F$. heteroclitus stratified by latitude and were distinct from each other (Figure $5 \mathrm{~b}$ ) and each $F$. heteroclitus population was clustered together (Figure 5c). F. grandis made its own cluster 
Table 3: Genetic parameters of sampled populations in two species of Fundulus.

\begin{tabular}{|c|c|c|c|c|c|}
\hline \multicolumn{6}{|l|}{ Fundulus heteroclitus } \\
\hline Population & PO & HO & HE & $\mathbf{F}$ & \% Departure from HWE \\
\hline Maine & 33 & 0.08 & 0.09 & $0.13+$ & 7.0 \\
\hline Sandwich & 48 & 0.12 & 0.14 & $0.13+$ & 9.3 \\
\hline New Bedford Harbor & 57 & 0.13 & 0.15 & $0.12 \dagger$ & 7.9 \\
\hline Point Judith & 44 & 0.11 & 0.13 & $0.18+$ & 10.8 \\
\hline Clinton & 59 & 0.12 & 0.13 & $0.09+$ & 6.0 \\
\hline Newark & 65 & 0.17 & 0.19 & $0.11 \dagger$ & 6.4 \\
\hline Tuckerton & 67 & 0.16 & 0.21 & $0.25 \dagger$ & 12.5 \\
\hline Magotha & 66 & 0.17 & 0.20 & $0.17 \dagger$ & 11.5 \\
\hline Elizabeth River & 67 & 0.16 & 0.20 & $0.23 \dagger$ & 12.3 \\
\hline Manteo & 65 & 0.16 & 0.20 & $0.19+$ & 12.9 \\
\hline Georgia & 51 & 0.13 & 0.16 & $0.19+$ & 13.2 \\
\hline Mean & 56.54 & 0.14 & 0.16 & 0.16 & 9.98 \\
\hline Standard Deviation & 11.28 & 0.03 & 0.04 & 0.05 & 2.76 \\
\hline \multicolumn{6}{|l|}{ Fundulus grandis } \\
\hline Population & Po & HO & HE & $\mathbf{F}$ & \% Departure from HWE \\
\hline Weeks Bay & 9.0 & 0.016 & 0.019 & $0.13 \dagger$ & 1.1 \\
\hline Dauphin Island & 5.9 & 0.016 & 0.024 & $0.23+$ & 2.8 \\
\hline Leeville & 5.9 & 0.017 & 0.020 & $0.32 \dagger$ & 2.0 \\
\hline Hackberry & 10.1 & 0.023 & 0.032 & 0.10 & 3.0 \\
\hline Port O'Connor & 8.5 & 0.018 & 0.026 & $0.27 \dagger$ & 4.2 \\
\hline Port Aransas & 3.7 & 0.021 & 0.031 & $0.23 \dagger$ & 2.1 \\
\hline Mean & 7.18 & 0.02 & 0.03 & 0.20 & 2.53 \\
\hline Standard Deviation & 2.4 & 0.003 & 0.005 & 0.11 & 1.06 \\
\hline
\end{tabular}

$+p \leq 0.01$ based on 10,000 permutations between individuals within the same populations.

and F. majalis and F. similis clustered together apart from other species (Figure $5 b$ ).

In $F$. heteroclitus, AMOVA showed that most of the variation was distributed within populations (59.05\%), but another large proportion of variation $(31.1 \%)$ was distributed among northern and southern regions. The remaining $9.85 \%$ of variation was explained by differences among populations within regions. In $F$. grandis, most of the variation was distributed within populations (82.4\%), and a smaller proportion (17.6\%) of variation was distributed longitudinally between populations across the Gulf of Mexico.
A Mantel test showed significant isolation by distance among $F$. heteroclitus populations $(\mathrm{p}<0.001)$ and $F$. grandis populations $(\mathrm{p}=0.032)$.

\section{Discussion}

We used high throughput sequencing and genotyping technology to identify and verify SNP markers in four non-model species within the Fundulus genus. Genotype data sharply differentiated northern and southern populations of $F$. heteroclitus as well as other species in this genus ( $F$. grandis, F. majalis, and $F$. similis). Within the species where SNPs were originally annotated, most can 
be successfully verified and used to study population structure as well as the role and outcome of selection forces on a genome-wide scale.

Using the 454 FLX pyrosequencing system, we observed 111,001 reads yielding an average of $22 \times$ coverage across 1,464 contigs. Read lengths and quality scores were similar to many other studies using the 454 FLX system to sequence uncharacterized genomes [8,70], but we identified fewer SNPs. Two-hundred and sixty-one SNPs were identified in 96 of these contigs (81 were further verified with the Sequenom MassARRAY platform). The percentage of contigs containing SNPs did differ between experiments: we obtained $0.07 \%$ of contigs containing SNPs while pyrosequencing of Eucalyptus ESTs identified $0.05 \%$ of contigs containing SNPs [8] and pyrosequencing of size selected, genomic DNA from swine identified $11.4 \%$ of contigs contained SNPs [70].
Our 454 pyrosequencing of genomic DNA was originally designed to both discover and genotype SNPs within and among populations of $F$. heteroclitus. Thus, we attempted to perform genome reduction with selective PCR reactions to approximately 200 loci that could be sequenced in 10 populations of 8 individuals. With 30,000 reads per one-eighth of a 454 sequencing plate, each region would have $15 \times$ coverage per individual or $980 \times$ coverage across all populations, enabling accurate genotype calls for most individuals. However, preselective amplication was not perfect, and many more than 200 loci were sequenced; most amplified only a single time in a single individual (these singlets therefore were not useful for variant detection). Furthermore, we obtained only $46 \%$ of the expected number of reads. In the end, these problems led to the inability to directly call individual genotypes. We were hoping to both identify SNPs and genotype individuals in a single step, but a more success-

(A)

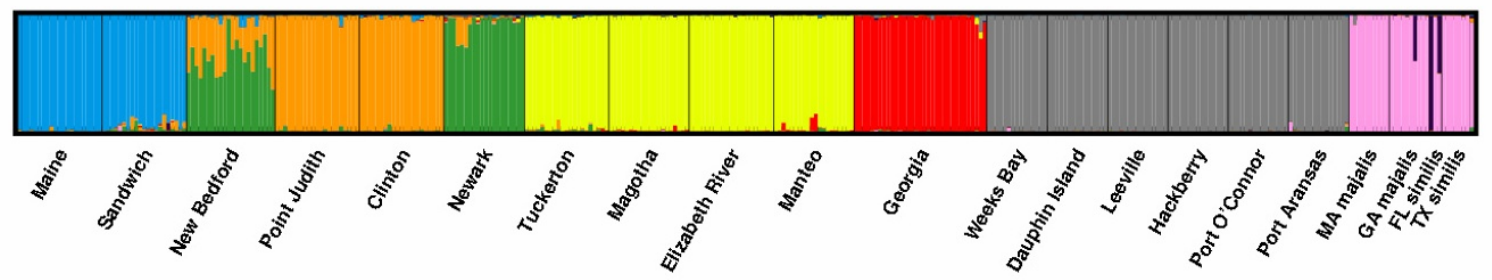

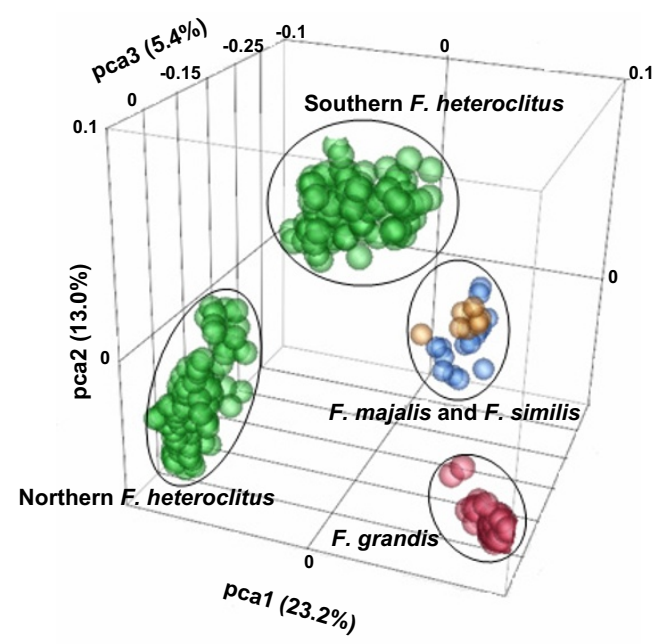

(B)

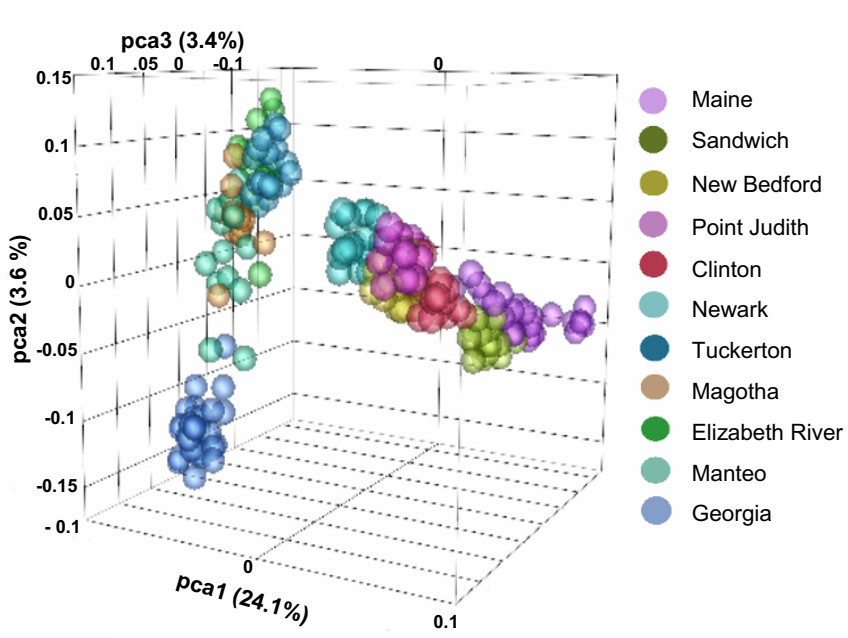

(C)

Figure 5 Structure of Fundulus populations. (A) Population structure as assessed by STRUCTURE. Bar plot was generated by DISTRUCT and depicts the classifications of the populations with the highest probability under the model. K indicates the number of clusters that maximized the probability of the model. Each individual is shown as a vertical bar. (B) Principal components PC1, PC2 and PC3 from all SNPs (as calculated in JMP Genomics 3.2) among all individuals. Species are separated from each other as well as northern and southern $F$. heteroclitus populations. Colors represent different species. (C) Principal components PC1, PC2, and PC3 from all SNPs among F. heteroclitus individuals. Colors represent different populations. 
ful approach (as evidenced by the swine group [70]) is to make reduced representation libraries from many pooled individuals for SNP discovery followed by individual genotyping. Because a pool of individuals is used, this approach identifies few singlets and thus enhances the number of reads per contig. Furthermore, improvements in both the number and length of reads using the Titanium series FLX 454 system compared to the original FLX system we used will increase the number of identified SNPs.

To increase our ability to measure population genetic parameters within and among populations, we verified SNPs identified through 454 sequencing and additional SNPs annotated from $F$. heteroclitus cDNAs using the MassARRAY system. Similar percentages of 454 pyrosequencing derived SNPs and SNPs identified from ESTs were verified ( $80 \%$ and $83 \%$, respectively). Of the 458 putative SNPs, 379 (82.75\%) were polymorphic, but only 264 had a greater than $90 \%$ successful call rate among all individuals. Among F. heteroclitus, most SNPs amplified (61.3\% were called in $>95 \%$ of individuals) indicating that differences in amplification rate between species led to the lower overall call rate. In white spruce, 91\% of SNPs verified with the Illumina SNP bead array platform $[71,72]$ were true. Comparable to F. heteroclitus, $70 \%$ of SNPs in spruce were called in greater than $95 \%$ of individuals [52]. Overall, verification of SNPs was powerful in providing information over many markers and individuals and was able to provide data to determine differences within populations, between populations and between species.

Species differentiation was demonstrated using principle component analysis (PCA) as well as STRUCTURE analysis. Both analyses showed separation between $F$. heteroclitus, $F$. grandis and F. majalis and similis as well as population structure within F. heteroclitus (Figure 4). These analyses provided the most resolution (even among distinguishing populations) in F. heteroclitus because the SNPs were originally identified in this species (i.e., due to an ascertainment bias). PCA and STRUCTURE did not differentiate sister species, F. similis and $F$. majalis, from each other or establish population structure within these species. Small sample sizes ( 1 to 10 individuals per population), high levels of monomorphism (average of $28 \%$ of all SNPs), and the fact that only $10 \%$ of SNP alleles differed between these two species, decreased the power to detect such differences when analyzed in conjunction with $F$. heteroclitus and F. grandis. Population structure also was masked in $F$. grandis when data was analyzed with other species. However, when F. grandis individuals were analyzed separately, they also showed distinct population structure (data not shown). One other study has reported multiple fixed differences in mitochondrial sequences between $F$. heteroclitus and F. gran- dis [33], but no other study to date has evaluated differences at many loci between all four species used in this study.

Within $F$. heteroclitus and F. grandis species, withinpopulation fixation indices $\left(\mathrm{F}_{\mathrm{IS}}\right.$, averaged across all loci) ranged from 0.09 to 0.32 . Among F. heteroclitus, all populations had an overall significant deficiency of heterozygotes indicated by positive $\mathrm{F}_{\mathrm{IS}}$ values. In these populations, approximately $10 \%$ of loci had similarly very large $F_{I S}$ values $(>0.5)$ across populations causing the skew in the average $F_{I S}$ value for each population. Within a population, these loci were predominately homozygous for one allele with a complete absence of the heterozygote and one or a few individuals homozygous for the alternative allele. The loci which presented this pattern were called conservatively at both alleles by Sequenom software across all individuals indicating that genotyping error was not the main reason for this pattern.

Furthermore, all northern populations were predominately homozygous for one allele and all southern populations were predominately homozygous for the alternative allele indicating strong demographic patterns in the data. The same demographic pattern was not found in F. grandis. Among F. grandis populations, most (70\%) SNPs with high $\mathrm{F}_{\mathrm{IS}}$ values were different between populations. This is in contrast to $F$. heteroclitus populations where loci with high $\mathrm{F}_{\mathrm{IS}}$ values were shared across populations. Within any one $F$. grandis population, one allele was predominant as a homozygote with one or a few individuals with the alternative homozygote. The most parsimonious explanation is that there is undetected substructure.

SNPs in Hardy-Weinberg were shown to be moderately polymorphic (average of 60\%) in F. heteroclitus. In F. grandis, SNPs were shown to lack polymorphism (7.18\%). The higher percentage of monomorphic loci in F. grandis likely is due to ascertainment bias in SNP discovery caused by only using F. heteroclitus populations. Many of the monomorphic loci (24\%) represent fixed differences between $F$. heteroclitus and F. grandis. Thus, while SNP markers developed in $F$. heteroclitus are not necessarily polymorphic in other Fundulus species, they still can be used to differentiate $F$. heteroclitus from other species.

Among $F$. heteroclitus populations, genotype data revealed strong latitudinal clines between the Northern and Southern F. heteroclitus populations. PCA, STRUCTURE, $\mathrm{F}_{\mathrm{ST}}$ values, and the isolation by distance test identified that individuals from Northern populations (above $40-41^{\circ} \mathrm{N}$ ) were distinct from Southern populations. This split is centered around the southern-most extent of the Atlantic coastal advancement during the late Pleistocene [73]. Specifically, observed heterozygosity and allelic richness across all loci is significantly lower $(\mathrm{p}=0.043, \mathrm{p}$ $=0.042$, respectively) in the north than in the south. 
These differences have been shown previously in morphological features [74] numerous allozyme loci [3436,75] and microsatellites [28]. The larger historical population size of $F$. heteroclitus in the south [28] would maintain greater heterozygosity and allelic richness at shared loci; in the north, where population sizes are smaller, loci have a higher probability of becoming fixed.

Four STRUCTURE clusters encompass the six northern populations while only two clusters encompass the five southern populations (Figure 5A). Separate northern clusters may be driven by smaller population sizes in which drift is greater. When genetic drift has a larger effect it becomes easier to distinguish populations because the average difference in allele frequencies of a marker in different populations will be greater. This is illustrated by a larger average $\mathrm{F}_{\mathrm{ST}}$ of 0.20 among northern populations in comparison to that of an average $\mathrm{F}_{\mathrm{ST}}$ of 0.10 among southern populations. This statistic is also evident for the north and south split, where populations from respective regions had an extremely high $\mathrm{F}_{\mathrm{ST}}$ value of 0.44 when compared against one another. Similar genetic divergence has been reported for $F$. heteroclitus using microsatellites ( 0.196 among northern populations, 0.117 among southern populations and 0.330 for the two most divergent populations, Nova Scotia and Georgia [28]). Similar demographic patterns have been described in freshwater fish [76] and marine species such as goby [77] and blue crab [78], and, as in Fundulus, these patterns are attributed to Pleistocene events.

A similar latitudinal cline occurs between populations of $F$. grandis, and a Mantel test shows significant isolation by distance. However, there were no significant differences between either levels of polymorphism or observed heterozygosity along latitude or longitude. Williams et al., 2008 reported significant isolation by distance as well as decreased allelic richness with increasing latitude. In this 2008 study, microsatellites were used, and two additional sites southern to those used in our study were included. Since microsatellites have many more alleles than SNPs and two additional sites were found to have relatively higher allelic richness in comparison to all other sampling sites along the gulf, this may account for the differences found in levels of polymorphism.

\section{Conclusions}

By targeting SNPs contained in both coding and noncoding areas of the genome, we are able to better understand how evolutionary forces are shaping the Fundulus genome. Similar studies using high throughput methods to sequence SNP markers have been developed in Atlantic cod [51], white spruce [52], Eucalyptus [8], and swine [70]. Like our study, these studies expanded their own species' knowledge base with respect to potential markers for studying evolutionary adaptation (in the case of cod and spruce), genome-wide assessment of diversity (Eucalyptus) or for use in breeding programs (swine)

\section{Additional material}

Additional file 1 SNP minor allele frequencies. Distributions of SNP minor allele frequencies (MAF) within F. heteroclitus and F. grandis populations.

\section{Authors' contributions}

LMW designed experiments, carried out laboratory and statistical analyses, and drafted the manuscript. XM carried out SNP detection of 454 data. ARB carried out SNP detection of 454 data, assisted in statistical analyses of MassARRAY data, and provided comments on earlier versions of this manuscript. CDB assisted in designed experiments and help develop SNP detection software. MFO designed experiments, assisted on statistical analysis, and helped to draft the manuscript. All authors read and approved the final manuscript

\section{Acknowledgements}

The authors thank G. Bozinovic and M. Everett for assistance in the collection of samples and D. Crawford for valuable input into methodology. Part of this work was carried out by using the resources of the Computational Biology Service Unit from Cornell University which is partially funded by Microsoft Corporation. Funding was partially provided by NIEHS Training Grant ES525163 award from the Department of Environmental and Molecular Toxicology at North Carolina State University to LMW, NIH RO1 ES011588 to MFO, NSF DEB0948510 to ARB, and NIH R01 HG003229 CDB.

\section{Author Details}

1Department of Environmental and Molecular Toxicology, Box 7633, North Carolina State University, Raleigh, NC 27695-7633, USA, 2Department of Biological Statistics and Computational Biology, Cornell University, Ithaca, NY 14853, USA and ${ }^{3}$ Rosenstiel School of Marine and Atmospheric Sciences, University of Miami, 4600 Rickenbacker Causeway, Miami, FL 33149, USA

Received: 5 February 2010 Accepted: 30 April 2010

Published: 30 April 2010

\section{References}

1. Bordoni R, Bonnal R, Rizzi E, Carrera P, Benedetti S, Cremonesi L, Stenirri S, Colombo A, Montrasio C, Bonalumi S, et al:: Evaluation of human gene variant detection in amplicon pools by the GS-FLX parallel Pyrosequencer. Bmc Genomics 2008, 9:464.

2. Garber M, Zody MC, Arachchi HM, Berlin A, Gnerre S, Green LM, Lennon N, Nusbaum C: Closing gaps in the human genome using sequencing by synthesis. Genome Biology 2009, 10:R60

3. Ingman M, Gyllensten U: SNP frequency estimation using massively parallel sequencing of pooled DNA. European Journal of Human Genetics 2009, 17:383-386.

4. Turner EH, Lee CL, Ng SB, Nickerson DA, Shendure J: Massively parallel exon capture and library-free resequencing across 16 genomes. Nature Methods 2009, 6:315-316.

5. Zheng JB, Moorhead M, Weng L, Siddiqui F, Carlton VEH, Ireland JS, Lee L, Peterson J, Wilkins J, Lin S, et al:: High-throughput, high-accuracy arraybased resequencing. Proceedings of the National Academy of Sciences of the United States of America 2009, 106:6712-6717.

6. De Schutter K, Lin YC, Tiels P, Van Hecke A, Glinka S, Weber-Lehmann J, Rouze P, de Peer YV, Callewaert N: Genome sequence of the recombinant protein production host Pichia pastoris. Nature Biotechnology 2009, 27:561-U104.

7. lacono M, Villa L, Fortini D, Bordoni R, Imperi F, Bonnal RJP, SicheritzPonten T, De Bellis G, Visca P, Cassone A, Carattoli A: Whole-genome pyrosequencing of an epidemic multidrug-resistant Acinetobacter baumannii strain belonging to the European clone II group. Antimicrobial Agents and Chemotherapy 2008, 52:2616-2625.

8. Novaes E, Drost DR, Farmerie WG, Pappas GJ, Grattapaglia D, Sederoff RR, Kirst M: High-throughput gene and SNP discovery in Eucalyptus grandis, an uncharacterized genome. Bmc Genomics 2008, 9: 
9. Vera JC, Wheat CW, Fescemyer HW, Frilander MJ, Crawford DL, Hanski I, Marden JH: Rapid transcriptome characterization for a nonmodel organism using 454 pyrosequencing. Molecular Ecology 2008, 17:1636-1647

10. Bontell IL, Hall N, Ashelford KE, Dubey JP, Boyle JP, Lindh J, Smith JE: Whole genome sequencing of a natural recombinant Toxoplasma gondii strain reveals chromosome sorting and local allelic variants. Genome Biology 2009, 10:R53.

11. Worden AZ, Panaud, Piegu : Green evolution and dynamic adaptation revealed by genomes of the marine picoeukaryotes Micromonas (vol 324, pg 268, 2009). Science 2009, 325:147-147.

12. Luikart G, England PR, Tallmon D, Jordan S, Taberlet P: The power and promise of population genomics: From genotyping to genome typing. Nature Reviews Genetics 2003, 4:981-994.

13. Diaz RJ: Overview of hypoxia around the world. Journal of Environmental Quality 2001, 30:275-281.

14. Diaz RJ, Rosenberg R: Marine benthic hypoxia: A review of its ecological effects and the behavioural responses of benthic macrofauna. Oceanography and Marine Biology - an Annual Review 1995, 33:245-303.

15. Smith KJ, Able KW: Dissolved oxygen dynamics in salt marsh pools and its potential impacts on fish assemblages. Marine Ecology-Progress Series 2003, 258:223-232.

16. Wirgin I, Waldman JR: Resistance to contaminants in North American fish populations. Mutation Research-Fundamental and Molecular Mechanisms of Mutagenesis 2004, 552:73-100.

17. Oleksiak MF, Churchill GA, Crawford DL: Variation in gene expression within and among natural populations. Nature Genetics 2002, 32:261-266

18. Oleksiak MF, Roach JL, Crawford DL: Natural variation in cardiac metabolism and gene expression in Fundulus heteroclitus. Nature Genetics 2005, 37:67-72.

19. Peterson JSK, Bain LJ: Differential gene expression in anthraceneexposed mummichogs (Fundulus heteroclitus). Aquatic Toxicology 2004, 66:345-355

20. Meyer JN, Volz DC, Freedman JH, Di Giulio RT: Differential display of hepatic mRNA from killifish (Fundulus heteroclitus) inhabiting a Superfund estuary. Aquatic Toxicology 2005, 73:327-341.

21. Whitehead A, Crawford DL: Variation in tissue-specific gene expression among natural populations. Genome Biology 2005, 6:R13.

22. Gonzalez HO, Roling JA, Baldwin WS, Bain LJ: Physiological changes and differential gene expression in mummichogs (Fundulus heteroclitus) exposed to arsenic. Aquatic Toxicology 2006, 77:43-52.

23. Roling JA, Bain LJ, Gardea-Torresdey J, Bader J, Baldwin WS: Hexavalent chromium reduces larval growth and alters gene expression in mummichog (Fundulus heteroclitus). Environmental Toxicology and Chemistry 2006, 25:2725-2733.

24. Whitehead A, Crawford DL: Neutral and adaptive variation in gene expression. Proceedings of the National Academy of Sciences of the United States of America 2006, 103:5425-5430.

25. Fisher MA, Oleksiak MF: Convergence and divergence in gene expression among natural populations exposed to pollution. BmC Genomics 2007, 8:108

26. Oleksiak MF: Changes in gene expression due to chronic exposure to environmental pollutants. Aquatic Toxicology 2008, 90:161-171.

27. Paschall JE, Oleksiak MF, VanWye JD, Roach JL, Whitehead JA, Wyckoff GJ, Kolell KJ, Crawford DL: FunnyBase: a systems level functional annotation of Fundulus ESTs for the analysis of gene expression. Bmc Genomics 2004, 5:96.

28. Adams SM, Lindmeier JB, Duvernell DD: Microsatellite analysis of the phylogeography, Pleistocene history and secondary contact hypotheses for the killifish, Fundulus heteroclitus. Molecular Ecology 2006, 15:1109-1123.

29. Bernardi G, Sordino P, Powers DA: Concordant mitochondrial and nuclear-DNA phylogenies for populations of the teloest fish Fundulus heteroclitus. Proceedings of the National Academy of Sciences of the United States of America 1993, 90:9271-9274.

30. Crawford DL, Powers DA: Evolutionary Adaptation to Different Thermal Environments Via Transcriptional Regulation. Molecular Biology and Evolution 1992, 9:806-813.

31. McMillan AM, Bagley MJ, Jackson SA, Nacci DE: Genetic diversity and structure of an estuarine fish (Fundulus heteroclitus) indigenous to sites associated with a highly contaminated urban harbor. Ecotoxicology 2006, 15:539-548.

32. Williams LM, Oleksiak MF: Signatures of selection in natural populations adapted to chronic pollution. Bmc Evolutionary Biology 2008, 8:282.

33. Whitehead A: Comparative mitochondrial genomics within and among species of killifish. Bmc Evolutionary Biology 2009, 9:11.

34. Powers DA, Place AR: Biochemical genetics of Fundulus heteroclitus ( $L$ ). Temporal and spatial variation in gene-frequencies of $L D H-B, M D H-A$, GPI-B, and PGM-A. Biochemical Genetics 1978, 16:593-607.

35. Powers DA, Ropson I, Brown DC, Vanbeneden R, Cashon R, Gonzalezvillasenor LI, Dimichele JA: Genetic variation in Fundulus heteroclitus - geographic distribution. American Zoologist 1986, 26:131-144.

36. Cashon RE, Vanbeneden RJ, Powers DA: Biochemical genetics of Fundulus heteroclitus ( $\mathrm{L}$ ). Spatial variation in gene-frequencies of IDH-A, IDH-B, 6-PGDH-A, and EST-S. Biochemical Genetics 1981, 19:715-728.

37. Crawford DL, Powers DA: Molecular-Basis of Evolutionary Adaptation at the Lactate Dehydrogenase-B Locus in the Fish Fundulus-Heteroclitus. Proceedings of the National Academy of Sciences of the United States of America 1989, 86:9365-9369.

38. Crawford DL, Constantino HR, Powers DA: Lactate Dehydrogenase-B Cdna from the Teleost Fundulus-Heteroclitus - Evolutionary Implications. Molecular Biology and Evolution 1989, 6:369-383.

39. Gill P: An assessment of the utility of single nucleotide polymorphisms (SNPs) for forensic purposes. International Journal of Legal Medicine 2001, 114:204-210.

40. Weir BS, Cardon LR, Anderson AD, Nielsen DM, Hill WG: Measures of human population structure show heterogeneity among genomic regions. Genome Research 2005, 15:1468-1476.

41. Paschou P, Ziv E, Burchard EG, Choudhry S, Rodriguez-Cintron W, Mahoney MW, Drineas P: PCA-correlated SNPs for structure identification in worldwide human populations. Plos Genetics 2007 3:1672-1686.

42. Yamaguchi-Kabata Y, Nakazono K, Takahashi A, Saito S, Hosono N, Kubo M, Nakamura Y, Kamatani N: Japanese Population Structure, Based on SNP Genotypes from 7003 Individuals Compared to Other Ethnic Groups: Effects on Population- Based Association Studies. American Journal of Human Genetics 2008, 83:445-456.

43. Primmer CR, Borge T, Lindell J, Saetre GP: Single-nucleotide polymorphism characterization in species with limited available sequence information: high nucleotide diversity revealed in the avian genome. Molecular Ecology 2002, 11:603-612.

44. Kong FR, Tong ZS, Chen XY, Sorrell T, Wang B, Wu QX, Ellis D, Chen S: Rapid identification and differentiation of Ttichophyton species, based on sequence Polymorphisms of the ribosomal internal transcribed spleacer regions, by rollingcircle amplification. Journal of Clinical Microbiology 2008, 46:1192-1199.

45. Johnson N, Fletcher O, Palles C, Rudd M, Webb E, Sellick G, Silva IDS, McCormack V, Gibson L, Fraser A, et al:: Counting potentially functional variants in BRCA1, BRCA2 and ATM predicts breast cancer susceptibility. Human Molecular Genetics 2007, 16:1051-1057.

46. Tomlinson I, Webb E, Carvajal-Carmona L, Broderick P, Kemp Z, Spain S, Penegar S, Chandler I, Gorman M, Wood W, et al: A genome-wide association scan of tag SNPs identifies a susceptibility variant for colorectal cancer at 8q24.21. Nature Genetics 2007, 39:984-988.

47. Amos Cl, Wu XF, Broderick P, Gorlov IP, Gu J, Eisen T, Dong Q, Zhang Q, Gu XJ, Vijayakrishnan J, et al.: Genome-wide association scan of tag SNPs identifies a susceptibility locus for lung cancer at 15q25.1. Nature Genetics 2008, 40:616-622

48. Poehlmann A, Kuester D, Meyer F, Lippert H, Roessner A, Schneider-Stock $\mathrm{R}$ : K-ras mutation detection in colorectal cancer using the Pyrosequencing technique. Pathology Research and Practice 2007, 203:489-497.

49. Mauricio R, Stahl EA, Korves T, Tian DC, Kreitman M, Bergelson J: Natural selection for polymorphism in the disease resistance gene Rps2 of Arabidopsis thaliana. Genetics 2003, 163:735-746.

50. Hoekstra HE, Hirschmann RJ, Bundey RA, Insel PA, Crossland JP: A single amino acid mutation contributes to adaptive beach mouse color pattern. Science 2006, 313:101-104.

51. Moen T, Hayes B, Nilsen F, Delghandi M, Fjalestad KT, Fevolden SE, Berg $P R$, Lien S: Identification and characterisation of novel SNP markers in Atlantic cod: Evidence for directional selection. Bmc Genetics 2008, 9:18. 
52. Namroud MC, Beaulieu J, Juge N, Laroche J, Bousquet J: Scanning the genome for gene single nucleotide polymorphisms involved in adaptive population differentiation in white spruce. Molecular Ecology 2008, 17:3599-3613.

53. Vignal A, Milan D, SanCristobal M, Eggen A: A review on SNP and other types of molecular markers and their use in animal genetics. Genetics Selection Evolution 2002, 34:275-305.

54. Wirgin II, Damore M, Grunwald C, Goldman A, Garte SJ: Genetic Diversity at an Oncogene Locus and in Mitochondrial-DNA between Populations of Cancer-Prone Atlantic Tomcod. Biochemical Genetics 1990, 28:459-475.

55. Aljanabi SM, Martinez I: Universal and rapid salt-extraction of high quality genomic DNA for PCR-based techniques. Nucleic Acids Research 1997, 25:4692-4693.

56. Parameswaran P, Jalili R, Tao L, Shokralla S, Gharizadeh B, Ronaghi M, Fire $A Z$ : A pyrosequencing-tailored nucleotide barcode design unveils opportunities for largescale sample multiplexing. Nucleic Acids Research 2007, 35:e130.

57. Margulies M, Egholm M, Altman WE, Attiya S, Bader JS, Bemben LA, Berka J, Braverman MS, Chen YJ, Chen ZT, et al:: Genome sequencing in microfabricated highdensity picolitre reactors. Nature 2005, 437:376-380.

58. Meyer M, Stenzel U, Hofreiter M: Parallel tagged sequencing on the 454 platform. Nature Protocols 2008, 3:267-278.

59. Huse SM, Huber JA, Morrison HG, Sogin ML, Welch DM: Accuracy and quality of massively parallel DNA pyrosequencing. Genome Biology 2007, 8:R143.

60. Brockman W, Alvarez P, Young S, Garber M, Giannoukos G, Lee WL, Russ C, Lander ES, Nusbaum C, Jaffe DB: Quality scores and SNP detection in sequencing-by-synthesis systems. Genome Research 2008, 18:763-770.

61. Huang $X Q$, Madan A: CAP3: A DNA sequence assembly program. Genome Research 1999, 9:868-877.

62. Batzoglou S, Jaffe DB, Stanley K, Butler J, Gnerre S, Mauceli E, Berger B, Mesirov JP, Lander ES: ARACHNE: A whole-genome shotgun assembler. Genome Research 2002, 12:177-189.

63. Duvernell DD, Lindmeier JB, Faust KE, Whitehead A: Relative influences of historical and contemporary forces shaping the distribution of genetic variation in the Atlantic killifish, Fundulus heteroclitus. Molecular Ecology 2008, 17:1344-1360.

64. Quackenbush J, Liang F, Holt I, Pertea G, Upton J: The TIGR Gene Indices: reconstruction and representation of expressed gene sequences. Nucleic Acids Research 2000, 28:141-145.

65. Hahn ME, Karchner SI, Franks DG, Merson RR: Aryl hydrocarbon receptor polymorphisms and dioxin resistance in Atlantic killifish (Fundulus heteroclitus). Pharmacogenetics 2004, 14:131-143.

66. Excoffier L, Laval G, Schneider S: Arlequin (version 3.0): An integrated software package for population genetics data analysis. Evolutionary Bioinformatics 2005:47-50.

67. Pritchard JK, Stephens M, Donnelly P: Inference of population structure using multilocus genotype data. Genetics 2000, 155:945-959.

68. Falush D, Stephens M, Pritchard JK: Inference of population structure using multilocus genotype data: Linked loci and correlated allele frequencies. Genetics 2003, 164:1567-1587.

69. Rosenberg NA: DISTRUCT: a program for the graphical display of population structure. Molecular Ecology Notes 2004, 4:137-138.

70. Wiedmann RT, Smith TPL, Nonneman DJ: SNP discovery in swine by reduced representation and high throughput pyrosequencing. BmC Genetics 2008, 9:81.

71. Shen R, Fan JB, Campbell D, Chang WH, Chen J, Doucet D, Yeakley J, Bibikova M, Garcia EW, McBride C, et al:: High-throughput SNP genotyping on universal bead arrays. Mutation Research-Fundamental and Molecular Mechanisms of Mutagenesis 2005, 573:70-82.

72. Fan J-B, Oliphant A, Shen R, Kermani BG, Garcia F, Gunderson KL, Hansen M, Steemers F, Butler SL, Deloukas P, et al.: Highly Parallel SNP Genotyping. Cold Spring Harbor Symposia on Quantitative Biology 2003, 68:69-78.

73. Mickelson DMCL, Fullerton DS, Borns HW, (Ed): Late-Quaternary Environments of the United States. Minneapolis: University of Minnesota Press; 1983.

74. Able KW, Felley JD: Geographical variation in Fundulus heteroclitus Tests for concordance between egg and adult morphologies. American Zoologist 1986, 26:145-157.
75. Ropson IJ, Brown DC, Powers DA: Biochemical genetics of Fundulus heteroclitus (L.) 6. Geographical variation in the gene frequencies of 15 loci. National Geographic Research 1990, 44:16-26.

76. Bernatchez L, Wilson CC: Comparative phylogeography of nearctic and palearctic fishes. Molecular Ecology 1998, 7:431-452

77. Gysels ES, Hellemans B, Pampoulie C, Volckaert FAM: Phylogeography of the common goby, Pomatoschistus microps, with particular emphasis on the colonization of the Mediterranean and the North Sea. Molecular Ecology 2004, 13:403-417.

78. McMillen-Jackson AL, Bert TM: Mitochondrial DNA variation and population genetic structure of the blue crab Callinectes sapidus in the eastern United States. Marine Biology 2004, 145:769-777.

doi: $10.1186 / 1471-2156-11-32$

Cite this article as: Williams et al., SNP identification, verification, and utility for population genetics in a non-model genus BMC Genetics 2010, 11:32

\section{Submit your next manuscript to BioMed Central and take full advantage of:}

- Convenient online submission

- Thorough peer review

- No space constraints or color figure charges

- Immediate publication on acceptance

- Inclusion in PubMed, CAS, Scopus and Google Scholar

- Research which is freely available for redistribution

Submit your manuscript at www.biomedcentral.com/submit
C) Biomed Central 